\title{
Dose Modification of Imatinib by Monitoring the Level of BCR-ABL Transcript in Chronic Myelogenous Leukemia
}

\author{
Izumi Ishikawa, ${ }^{1}$ Chiaki Kato, ${ }^{2}$ Hideo Harigae, ${ }^{3}$ Tomohiro Sugawara, ${ }^{1}$ \\ Yasuo Tomiya, ${ }^{3}$ Minami Yamada, ${ }^{3}$ Kenichi Ishizawa, ${ }^{3}$ Junichi Kameoka, \\ KoICHi MiYamura ${ }^{4}$ and TAKeShi $\mathrm{SASAKI}^{3}$ \\ ${ }^{I}$ Department of Internal Medicine, Osaki Citizen Hospital, Osaki, Japan \\ ${ }^{2}$ Division of Hematology, National Hospital Organization Nagoya Medical \\ Center, Nagoya, Japan \\ ${ }^{3}$ Department of Rheumatology and Hematology, Tohoku University Graduate \\ School of Medicine, Sendai, Japan \\ ${ }^{4}$ Department of Internal Medicine, Japanese Red Cross Nagoya First Hospital, \\ Nagoya, Japan
}

Ishikawa, I., Kato, C., Harigae, H., Sugawara, T., Tomiya, Y., Yamada, M., Ishizawa, K., Kameoka, J., MiYamura, K. and Sasaki, T. Dose Modification of Imatinib by Monitoring the Level of BCR-ABL Transcript in Chronic Myelogenous Leukemia. Tohoku J. Exp. Med., 2006, 210 (4), 355-363 — Chronic myelogenous leukemia (CML) is a hematological malignancy that is characterized by the chromosome anomaly, $t(9 ; 22)(\mathrm{q} 34 ; \mathrm{q} 11)$. By this chromosomal translocation, a novel activated tyrosine kinase, BCR-ABL chimeric protein, is generated, and the protein is causative of the disease. Recently, Imatinib mesylate targeting to a BCR-ABL chimeric protein has been developed, and shown to achieve complete remission at a high rate. Patients are currently required to receive a fixed dose, $400 \mathrm{mg}$ daily; however, it is possible that some of patients can maintain their remission with reduced doses of imatinib. In this study, we determined levels of BCR-ABL transcript in CML patients by real-time quantitative polymerase chain reaction analysis, and explored the possibility of individualization of therapeutic doses of imatinib. Thirty-five CML patients, including 17 newly diagnosed patients, 16 patients pre-treated with interferon-alpha, and 2 relapsed patients after allogeneic transplantation, were treated with imatinib. Complete cytogenetic response was achieved in $31(89 \%)$ patients. Major molecular response (MMR) was achieved in $21(60 \%)$. Complete molecular response (CMR) was achieved in $7(20 \%)$. Imatinib was discontinued in 2 patients, one patient with MMR due to noncompliance and other patient sustaining CMR, but both patients relapsed 7 and 13 months later, respectively. The doses of imatinib were reduced in 7 patients due to its side effects, but 4 out of the 7 patients have sustained MMR, and 2 of them have sustained CMR for more than 23 months. These results indicate that some patients are able to maintain MMR with low-dose imatinib. — chronic myeloid leukemia; imatinib mesylate; RQ-PCR; BCR-ABL; dose modification (C) 2006 Tohoku University Medical Press

Received August 30, 2006; revision accepted for publication October 18, 2006.

Correspondence: Izumi Ishikawa, M.D., Department of Internal Medicine, Osaki Citizen Hospital, Osaki 989-6183, Japan.

e-mail: idumi000@hotmail.com 
Chronic myelogenous leukemia (CML) is a lethal hematologic disorder that results from malignant transformation of a very primitive hematopoietic cell. chromosomal translocation, $\mathrm{t}(9 ; 22)(\mathrm{q} 34 ; \mathrm{q} 11)$, forms the Philadelphia $(\mathrm{Ph})$ chromosome and creates a novel fusion gene, BCR-ABL (Sawyers 1999). This fusion gene expresses an activated tyrosine kinase that is the cause of CML (Daley et al. 1990; Lugo et al. 1990). The median survival among patients with CML is three to six years, with most deaths resulting from progression of the disease into a blastic phase. Interferon-alpha (IFN) induced cytogenetic response, but median survival durations with IFN were 5 to 7 years. Although 10-year survival rate of patients who achieved complete cytogenetic response (CCyR) is $70 \%$ to $85 \%$, IFN induced a CCyR only in 5\% to $30 \%$ of patients (Kantarjian et al. 1995; Italian Cooperative Study Group 1999; Faderl et al. 1999; Bonifazi et al. 2001).

Imatinib mesylate has become the standard drug treatment for patients with CML (Kantarjian et al. 2002a; Talpaz et al. 2002; O'Brien et al. 2003). Imatinib is a selective inhibitor of the BcrAbl tyrosine kinase activity. This drug is thought to bind competitively to the adenosine triphosphate (ATP)-docking site of tyrosine kinase proteins, including $\mathrm{ABL}$ itself and the hybrid BCRABL proteins (Schindler et al. 2000). The International Randomized Study of IFN and STI571 (IRIS) trial demonstrated that imatinib treatment induced rapid and substantial reductions in the leukemic load. After 12 months of treatment, an estimated $39 \%$ of patients in the imatinib group had at least 3-log reduction of BCR-ABL/ BCR levels, as compared with $2 \%$ of patients in the IFN group, suggesting the superiority of imatinib to IFN in the treatment of CML. Moreover, the probability of patients who had at least 3-log in BCR-ABL/BCR levels progression-free was $100 \%$ at 24 months, as compared with $95 \%$ for patients who had a CCyR with a reduction of less than 3-log and $85 \%$ for patients who did not have a CCyR (Hughes et al. 2003). These lines of evidence indicate that imatinib is a standard drug in the treatment of CML and monitoring of minimal residual disease (MRD) by real-time quantitative PCR (RQ-PCR) is essential in the evaluation of disease status.

In this study, we used RQ-PCR to measure the level of BCR-ABL transcripts in patients with CML in chronic phase treated with imatinib. Based on the results, we determined the probability of achieving molecular remission and proposed the individualization of therapeutic doses of imatinib.

\section{Patients and Methods}

\section{Patients}

From December 2000 to August 2005, 35 patients with chronic phase CML were treated with imatinib: 17 were newly diagnosed patients in early chronic phase, 16 were IFN pre-treated patients in late chronic phase and 2 were relapsed patients after allogeneic stem cell transplantation (SCT) (one was a molecular relapse and the other was cytogenetic relapse).

The chronic phase was defined by the presence of less than $15 \%$ blasts, less than $20 \%$ basophils, and less than $30 \%$ blasts plus promyelocytes in the peripheral blood and bone marrow and a platelet count of at least 100,000 per cubic millimeter and no evidence of extramedullary disease (Kantarjian et al. 2002a, b; 2003). Molecular relapse was defined as (1) the BCR-ABL/ABL ratio is more than $0.02 \%$ on 3 consecutive occasions at least one month apart; (2) the ratio is more than $0.05 \%$ on 2 consecutive samples; or (3) the transcript number is rising in 3 consecutive samples, with the last 2 samples greater than $0.02 \%$ (Olavarria et al. 2001).

Patients received imatinib $400 \mathrm{mg}$ p.o. daily. The dose was adjusted according to non-hematological and hematological toxicities. Drug toxicity was evaluated at each visit and graded according to the National Cancer Institute Common Toxicity Criteria (version 2.0). Informed consent according to institutional regulations by the document or oral was obtained.

\section{Evaluation of patients}

Peripheral blood for RQ-PCR was collected at the before imatinib treatment and repeated every 3 to 6 months after treatment was started. Response criteria were the same as those used in published studies using imatinib (Kantarjian et al. 2002a; Talpaz et al. 2002). A complete hematologic response (CHR) was defined by a white blood cell count of less than $10,000 / \mu 1$, a platelet count of less than $450,000 / \mu 1$, the presence of less than 
$5 \%$ myelocytes and metamyelocytes and less than $20 \%$ basophils in peripheral blood, the absence of blasts and promyelocytes in peripheral blood, and disappearance of all signs and symptoms related to leukemia (including palpable splenomegaly) lasting for at least 4 weeks. Cytogenetic response was based on the prevalence of $\mathrm{Ph}$ metaphases among at least 20 metaphase cells in each bone marrow sample and was defined as complete $(0 \%$ $\mathrm{Ph}$-positive cells), partial (1\%-35\%), minor (36\%-65\%), minimal (66\%-95\%), none (>95\%). Major cytogenetic response (MCyR) was defined as either a complete or partial response. A major molecular response (MMR) was defined as a 3-log reduction in BCR-ABL transcript levels compared with that seen in samples before imatinib treatment (Hughes et al. 2003). A complete molecular response $(\mathrm{CMR})$ was defined as undetectable levels of BCR-ABL confirmed by nested PCR.

\section{Cytogenetic analysis, PCR}

Cytogenetic analysis was performed by the G-banding technique. Marrow specimens were examined on direct short-term (24-hr) cultures; at least 20 metaphases were analyzed. BCR-ABL transcript levels were determined by RQ-PCR analysis on peripheral blood. Nested PCR techniques were used to confirm the results in samples defined as having undetectable BCRABL levels by RQ-PCR. Total RNA from peripheral blood $(7 \mathrm{ml})$ was extracted by a guanidinium isothiocyanate-acid phenol procedure, and $4 \mu \mathrm{g}$ of total RNA was reverse-transcribed into cDNA with random hexamer primers (Perkin-Elmer, Wellesley, MA, USA). Finally, $80 \mu \mathrm{l}$ of cDNA solution was then stored at $-20^{\circ} \mathrm{C}$ until assay.

The RQ-PCR primers and the probe for BCR-ABL were as follows: BCR sense: 5'-TCC GCT GAC CAT CAA TAA GGA (corresponding to nt3173-3193); and ABL antisense: 5'-CAC TCA GAC CCT GAG GCT CAA (corresponding to nt 277-257). TaqMan probe for b3a2 and b2a2 was: CCC TTC AGC GGC CAG TAG CAT CTG A (corresponding to nt 230-254 of ABL gene). Primers and probe for ABL were: ABL sense, 5'-TGG AGA TAA CAC TCT AAG CAT AAC TAA AGG (corresponding to nt 372-401); ABL antisense, 5'-GAT GTA GTT GCT TGG GAC CCA (corresponding to $\mathrm{nt}$ 495-475); and TaqMan probe for ABL, 5'-CCA TTT TTG GTT TGG GCT TCA CAC CAT T (corresponding to nt 467-440). Sequences of primers and probes were kindly provided by Prof. J. Gabert (Gabert et al. 2003). Probes were labeled with 6-carboxy-fluorescein as the reporter dye and 5-carboxy-tetramethyl-rhodamine as the quencher fluorescent. Calibration for quantification of each transcript was obtained by using Fusion Quant Calibrators (Ipsogen, Marseille, France).

RQ-PCR was performed on an ABI PRISM 7700 Sequence detector (Applied Biosystems, Foster City, CA, USA). The 50- $\mu 1$ PCR reaction mix contained $5 \mu 1$ of 10 $\times$ PCR buffer, $5 \mathrm{mM} \mathrm{MgCl} 2,250 \mu \mathrm{M}$ dNTP, $200 \mathrm{nM}$ each primer, $100 \mathrm{nM}$ probe, 1.25 units of a temperaturerelease Taq DNA polymerase, and $4 \mu 1$ of sample cDNA. PCR amplification began with a 10-min denaturation step at $95^{\circ} \mathrm{C}$, followed by 40 cycles of denaturation at $95^{\circ} \mathrm{C}$ for 15 second and annealing/extension at $58^{\circ} \mathrm{C}$ for 60 second. ABL was used as the control gene and the BCRABL levels for each sample were expressed as a percentage of BCR-ABL to ABL. Our preliminary study using leukemic cell lines revealed the RQ-PCR was able to detect 1 BCR-ABL positive cells in $10^{5}$ of BCR-ABL negative cells. The median value calculating from 20 samples collected from patients with newly diagnosed chronic-phase CML who had not yet begun taking imatinib was used as the standard baseline. The reduction in BCR-ABL levels from the standard baseline value was calculated for each sample as described by Hughes et al. (2003).

\section{REsults}

\section{Patients}

The median follow up from the time treatment was started is 29 months (range, 9-38 months). The median age of the study group was 47.8 years (range, 21-77 years). Twenty-two patients were man and 13 were woman. CHR was achieved in 32 of $35(91 \%)$ patients (Fig. 1). These included 16 of $17(94 \%)$ newly-diagnosed patients in early chronic phase, 14 of $16(88 \%)$ IFN pre-treated patients in late chronic phase, and 2 relapsed patients after allogeneic SCT (100\%). All of 32 patients with CHR achieved MCyR (Fig. 1). CCyR was achieved in 31 of $35(89 \%)$ patients (Fig. 1); 16 of 17 (94\%) in early chronic phase and 13 of $16(81 \%)$ in late chronic phase. Three patients had no response. Though we evaluated for BCR-ABL kinase domain mutations by direct sequencing, mutation was not detected at the hot spot in 3 cases of no response to imatinib (data not shown). None have died while on therapy with imatinib, and transformation while on 
(A)

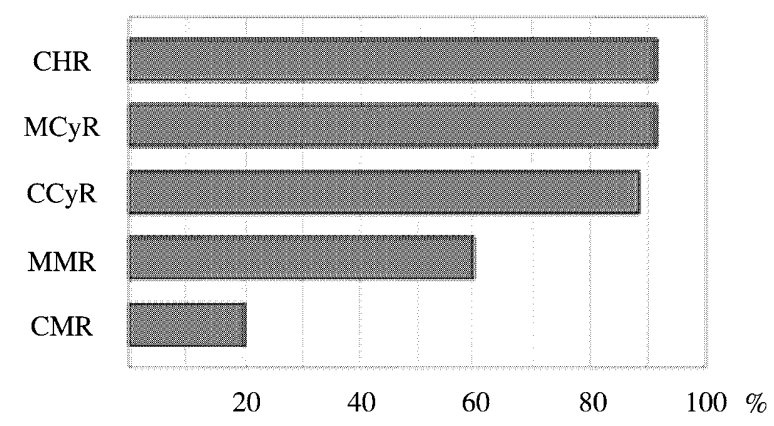

(B)

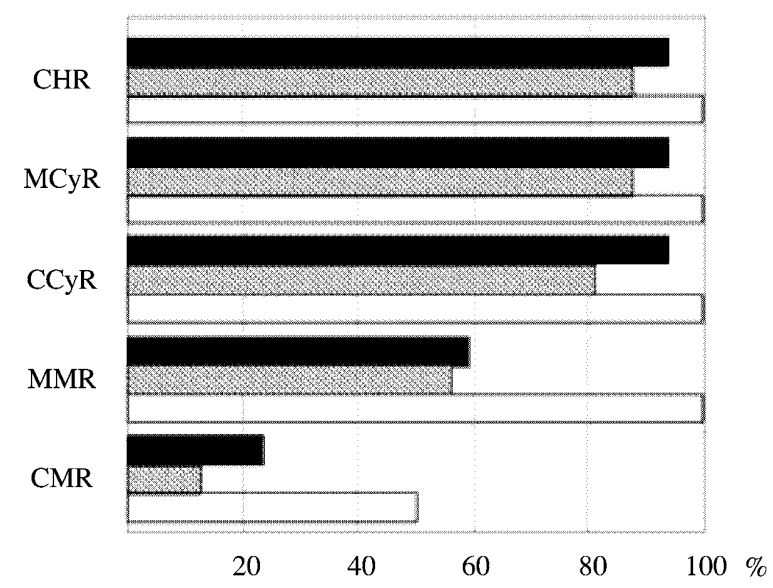

Fig. 1. Response to imatinib treatment.

Response to imatinib treatment of 35 patients with CML is shown. Response rate was represented by percentage of patients who fulfilled each of the response criteria. (A) all patients and (B) patients divided by clinical phase of CML. solid bar, early chronic phase; hatched bar, late chronic phase; open bar, relapse after allo-SCT.

CHR, complete hematologic response; MCyR, major cytogenetic response; $\mathrm{CCyR}$, complete cytogenetic response; MMR, major molecular response; CMR, complete molecular response.

therapy did not occur.

\section{Molecular response}

The median BCR-ABL/ABL ratio before imatinib treatment was $49.43 \%$ (range, $0.115-289.57 \%$ ). Therefore, a MMR was defined as less than the ratio of $0.049 \%$ represented a $3-\log$ reduction in this study.

As shown in Fig. 1, MMR was achieved in
21 of $35(60 \%)$ patients; 10 of $17(59 \%)$ in early chronic phase, 9 of $16(56 \%)$ in late chronic phase and 2 of $2(100 \%)$ in relapse after allogeneic SCT. MMR was sustained in 16 of $21(76 \%)$ patients, for a median of 13.5 months (range, 1-36 months). Five patients lost MMR after having therapy interrupted temporarily or permanently. The reasons for treatment interruptions were toxicity in four and noncompliance in one. Three of 5 patients achieved MMR again and 2 of 5 had a 2-log reduction by restarting imatinib therapy. CMR was achieved in 7 of 35 (20\%) patients; 4 of $17(24 \%)$ in early chronic phase, 2 of $16(13 \%)$ in late chronic phase and 1 of $2(50 \%)$ in relapse after allogeneic SCT.

\section{Toxicity}

Side-effects of imatinib were observed in 29 patients $(83 \%)$. Fluid retention, skin rash, nausea, diarrhea, and muscle cramps were common. Grade 3 or higher toxicity was observed in 15 patients $(43 \%)$. Grade 3 or higher nonhematologic adverse event was skin rash in 6 patients $(17 \%)$. All toxicities were reversible with dose reductions or treatment interruptions. Grade 3 or higher hematologic toxicity was neutropenia in 11 patients $(31 \%)$ and thrombocytopenia in 2 patients $(6 \%)$. Seven patients required dose reduction of imatinib for the side effect. The most common causes for dose reduction were myelosuppression, skin rash, fluid retention, and diarrhea.

\section{Dose modification}

Two patients discontinued imatinib. One patient, who relapsed in molecular level after allogeneic SCT, sustained CMR more than 6 months, and then discontinued imatinib after confirming CMR by nested RT-PCR (Fig. 2A). Seven months after discontinuation of therapy, the patient had a molecular relapse again. Imatinib was resumed at $400 \mathrm{mg}$, and 4 months later BCRABL was again undetectable. The other discontinued therapy after achieved major molecular remission due to noncompliance, and had a cytogenetic relapse 13 months later.

Imatinib dose was reduced in 7 patients. 
(A)

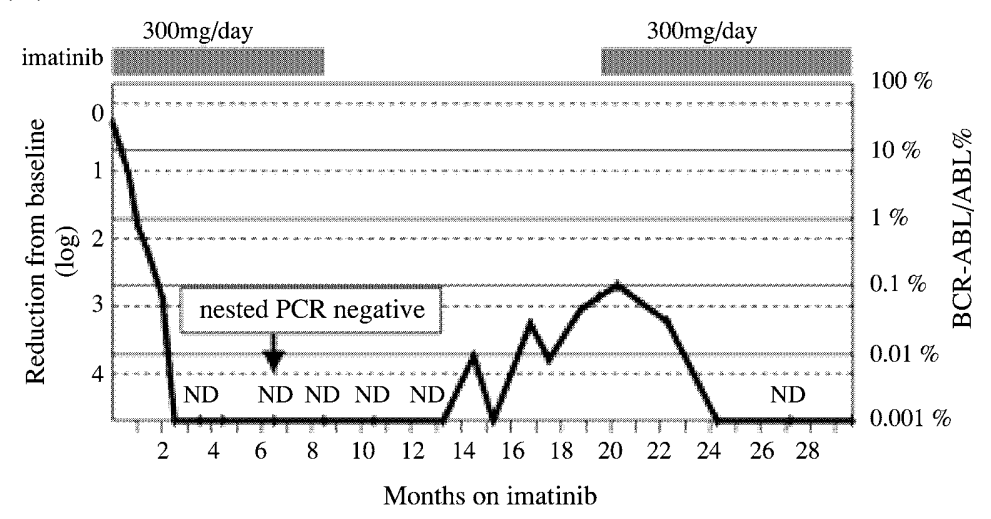

(B)

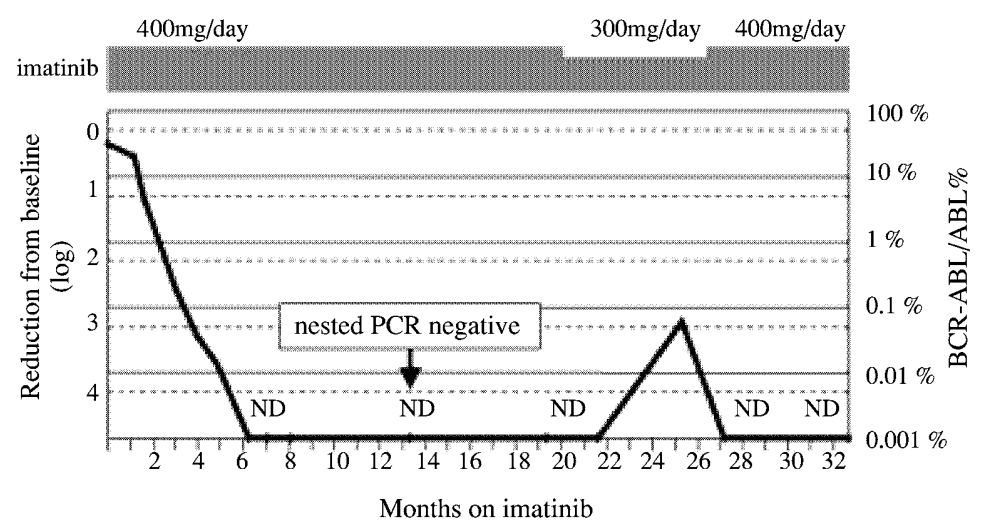

Fig. 2. Molecular relapse due to imatinib discontinuation or tapering.

Clinical courses of patients, who exhibited molecular relapse after discontinuation or tapering of imatinib, are shown. (A) A female patient with molecular relapse after allotransplant was treated with imatinib, and discontinued after 6 months of CMR period. The patient relapsed after 7 months. (B) A female patient in early chronic phase reduced the dose due to nonconpliance. The patient relapsed after 7 months.

After dose reduction of imatinib, BCR-ABL/ABL ratio increased to more than 2-log reduction level in 3 patients. They resumed imatinib at $400 \mathrm{mg}$ daily. One of 3 achieved a second CMR (Fig. 2B), and others achieved 2-log reduction later.

On the other hand, molecular relapse was not seen in 4 patients. They were treated with less than $300 \mathrm{mg} /$ day of imatinib because of the sideeffects. Interestingly, 2 cases achieved CMR with alternating daily doses of $200 \mathrm{mg}$ and $100 \mathrm{mg}$ (Fig. $3 \mathrm{~A}, \mathrm{~B})$. As of this writing, they are currently receiving lower dose of imatinib therapy and are still in CMR (23+ and 29+ months, respectively).

The dose was increased to $600 \mathrm{mg}$ daily in one patient because of poor molecular response.
The patient achieved a major molecular remission after the dose escalation of imatinib (data not shown).

\section{Discussion}

Since CCyR is achieved in $75 \%$ to $90 \%$ of CML patients treated with imatinib in early chronic phase (O'Brien et al. 2003; Kantarjian et al. 2003; 2004), the sensitivity of conventional chromosomal analysis is not satisfactory for evaluating the disease status. Recently, RQ-PCR targeting $\mathrm{BCR}-\mathrm{ABL}$ has been routinely used to monitor MRD in CML patients as well as $\mathrm{Ph}+$ ALL patients (Yamada et al. 2004).

The definition of MMR by RQ-PCR is argu- 
(A)

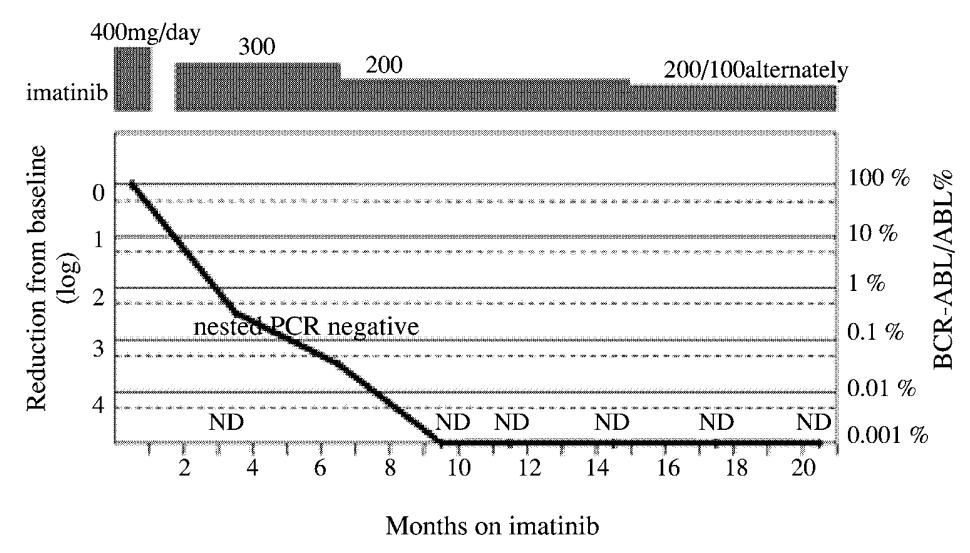

(B)

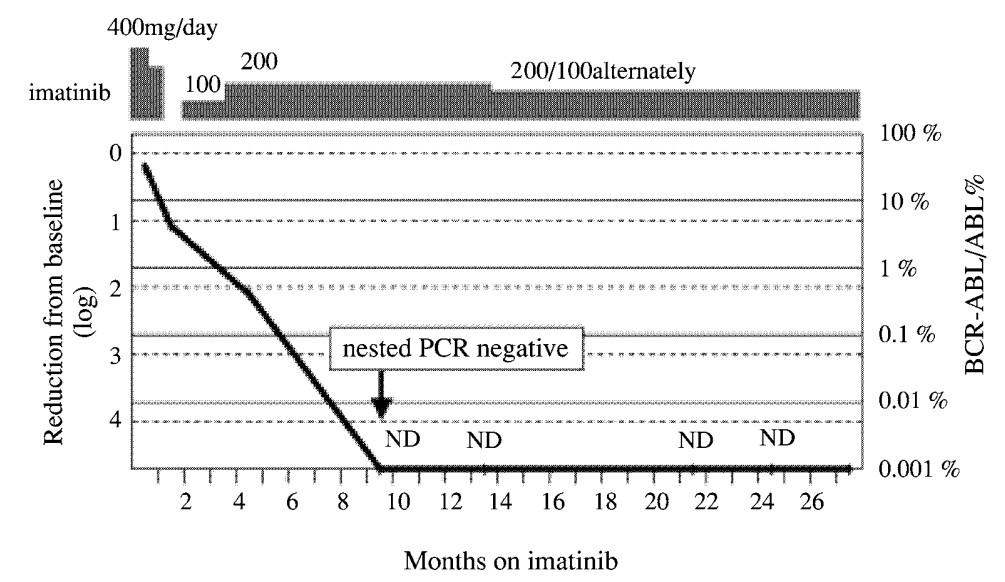

Fig. 3. Sustained molecular response despite imatinib tapering.

Clinical courses of patients, who sustained CMR after tapering of imatinib, are shown. (A) A male patient in early chronic phase resumed $300 \mathrm{mg}$ of imatinib after temporal cessation due to side effect, and the dose was reduced gradually. The patient sustained CMR at alternate doses of $100 \mathrm{mg}$ and $200 \mathrm{mg}$. (B) A patient in early chronic phase sustained CMR at alternate doses of $100 \mathrm{mg}$ and $200 \mathrm{mg}$.

$\mathrm{ND}$; not detected.

able. Hughes et al. (2003) defined 3-log reduction of BCR-ABL/ABL ratio from that before the start of therapy as MMR. The 3-log reduction has been shown to be associated with progression-free survival, however, this is not applicable for pretreated patients whose $\mathrm{BCR}-\mathrm{ABL} / \mathrm{ABL}$ ratio is already decreased. Another definition means an absolute value of BCR-ABL/ABL ratio is less than $0.05 \%$. This cutoff was based on previous studies identifying BCR-ABL/ABL ratios of $0.045 \%$ to $0.1 \%$ to be prognostically important (Hochhaus et al. 2000; Paschka et al. 2003).
According to these criteria, MMR has been shown to be achieved in $39 \%$ to $62 \%$, and $4 \%$ to $38 \%$ of patients have achieved undetectable levels of BCR-ABL (Hughes et al. 2003; O'Brien et al. 2003; Cortes et al. 2005). In this study, among 32 patients who achieved CCyR with imatinib, 21 (60\%) achieved MMR, and 7 (20\%) had CMR. It was likely that the patients in early chronic phase tend to achieve better response than those in late chronic phase, but there was no significant difference in follow-up between the groups. Cortes et al. (2005) reported that MMR has been sustained 
in $77 \%$ of patients, and CMR has been sustained in nearly half of the patients. Overall, our results were obtained from small population $(n=35)$, but those were consistent with the published reports.

Currently, $400 \mathrm{mg}$ per day of imatinib is given uniformly to all patients. It is possible some patients do not need to receive full dose of imatinib to keep remission; however, it is unclear whether imatinib therapy can be safely discontinued in patients with complete cytogenetic and molecular responses. To our knowledge, there are 11 other reported cases of imatinib discontinuation due to toxicity or patients' request: 9 had undetectable levels of BCR-ABL transcript (Cortes et al. 2004; Mauro et al. 2004; Merante et al. 2005) and 2 were in cytogenetic response (Ghanima et al. 2004; Ali et al. 2005). Overall, in 7 of the literature cases, molecular and cytogenetic relapse occurred rapidly 3 to 10 months after imatinib discontinuation. Thus, 7 of $11(64 \%)$ reported cases relapsed after withdrawal of imatinib. However, 3 patients were in CMR for 13 to 15 months, and one patient in MCR for 18 months without imatinib. In this study, imatinib was discontinued in 2 patients. At the time of imatinib withdrawal, one patient had been in sustained CMR for 18 months and the other in MMR for 3 months. Both patients relapsed after imatinib discontinuation (7 and 13 months later, respectively). Cortes et al. (2004) proposed it cannot be recommended at the present time to discontinue therapy for patients who achieve a molecular remission with imatinib. Bhatia et al. (2003) demonstrated that $\mathrm{BCR} / \mathrm{ABL}$ positive hematopoietic progenitors persist in the marrow of patients in CCyR, indicating that CML progenitors may be suppressed but not eliminated in the imatinib treatment. Probably, patients even in CMR should not be discontinued imatinib therapy at the present time, because most cases relapsed after withdrawal of imatinib therapy, suggesting that undetectable BCR-ABL transcript would not mean cure. However, a few cases remained in a molecular remission would imply the possibility of imatinib discontinuation for some patients.

As regards to dose modification, we presented suggestive cases in this study. In seven patients who required a dose reduction of imatinib, 3 patients had a molecular relapse but 4 patients sustained in MMR without increasing of BCRABL level. In addition, the 4 patients without molecular relapse were treated with imatinib less than $300 \mathrm{mg}$ daily due to side-effects. Although the follow-up of our patients is short, 2 of 4 patients are still in CMR with this lower dose of imatinib therapy for more than 23 months respectively, as of this writing. These results suggest that dose reduction, not discontinuation, may be acceptable in some patients in CMR. It should be highlighted that MRD monitoring is essential when dose reduction, because CML cells may obtain imatinib resistance at lower doses of imatinib (Yoshida and Melo 2004).

In conclusion, our results suggest that some patients with CML in MMR are able to be treated with low-dose imatinib, thus leading to reducing the cost of treatment. Further exploratory and comparative studies will be crucial to establish the safety of dose reduction of imatinib.

\section{Acknowledgments}

Authors thank Ms. M. Tada, Y. Suzuki, I. Osawa and M. Watanabe for their technical assistance.

\section{References}

Ali, R., Ozkalemkas, F., Ozcelik, T., Ozkocaman, V., Ozan, U., Kimya, Y., Koksal, N., Gulten, T., Yakut, T. \& Tunali, A. (2005) Pregnancy under treatment of imatinib and successful labor in a patient with chronic myelogenous leukemia (CML). Outcome of discontinuation of imatinib therapy after achieving a molecular remission. Leuk Res., 29, 971-973.

Bhatia, R., Holtz, M., Niu, N., Gray, R., Snyder, D.S., Sawyers, C.L., Arber, D.A., Slovak, M.L. \& Forman, S.J. (2003) Persistence of malignant hematopoietic progenitors in chronic myelogenous leukemia patients in complete cytogenetic remission following imatinib mesylate treatment. Blood, 101, 4701-4707.

Bonifazi, F., Vivo, A., Rosti, G., Guilhot, F., Guilhot, J., Trabacchi, E., Hehlmann, R., Hochhaus, A., Shepherd, P.C., Steegmann, J.L., Kluin-Nelemans, H.C., Thaler, J., Simonsson, B., Louwagie, A., Reiffers, J., Mahon, F.X., Montefusco, E., Alimena, G., Hasford, J., Richards, S., Saglio, G., Testoni, N., Martinelli, G., Tura, S. \& Baccarani, M.; Europena Study Group on Interferon in Chronic Myeloid Leukemia; Italian Cooperative Study Group on CML; France Intergroup of CML; German CML Study Group; UK Medical Research Council Working Party on CML; Spanish CML Study Group; Australian CML Study Group; Swedish CML Study Group. (2001) Chronic myeloid leukemia and interferon-alpha: a study of complete 
cytogenetic responders. Blood, 98, 3074-3081.

Cortes, J., O’Brien, S. \& Kantarjian, H. (2004) Discontinuation of imatinib therapy after achieving a molecular response. Blood, 104, 2204-2205.

Cortes, J., Talpaz, M., O’Brien, S., Jones, D., Luthra, R., Shan, J., Giles, F., Faderl, S., Verstovsek, S., Garcia-Manero, G., Rios, M.B. \& Kantarjian, H. (2005) Molecular Responses in Patients with Chronic Myelogenous Leukemia in Chronic Phase Treated with Imatinib Mesylate. Clin. Cancer Res., 11, 3425-3232.

Daley, G.Q., Etten, R.A. \& Baltimore, D. (1990) Induction of chronic myelogenous leukemia in mice by the P210bcr/abl gene of the Philadelphia chromosome. Science, 247, 824-830.

Faderl, S., Talpaz, M., Estrov, Z. \& Kantarjian, H.M. (1999) Chronic myelogenous leukemia; biology and therapy. Ann. Intern. Med., 131, 207-219.

Gabert, J., Beillard, E., Velden, V.H., Bi, W., Grimwade, D., Pallisgaard, N., Barbany, G., Cazzaniga, G., Cayuela, J.M., Cave, H., Pane, F., Aerts, J.L., De Micheli, D., Thirion, X., Pradel, V., Gonzalez, M., Viehmann, S., Malec, M., Saglio, G. \& Dongen, J.J. (2003) Standardization and quality control studies of 'real-time' quantitative reverse transcriptase polymerase chain reaction of fusion gene transcripts for residual disease detection in leukemia - a Europe Against Cancer program. Leukemia, 17, 2318-2357.

Ghanima, W., Kahrs, J., Dahl, T.G., III \& Tjonnfjord, G.E. (2004) Sustained cytogenetic response after discontinuation of imatinib mesylate in a patient with chronic myeloid leukaemia. Eur. J. Haematol., 72, 441-443.

Hochhaus, A., Reiter, A., Saussele, S., Reichert, A., Emig, M., Kaeda, J., Schultheis, B., Berger, U., Shepherd, P.C., Allan, N.C., Hehlmann, R., Goldman, J.M. \& Cross, N.C. (2000) Molecular heterogeneity in complete cytogenetic responders after interferon-alpha therapy for chronic myelogenous leukemia: low levels of minimal residual disease are associated with continuing remission. German CML Study Group and the UK MRC CML Study Group. Blood, 95, 62-66.

Hughes, T.P., Kaeda, J., Branford, S., Rudzki, Z., Hochhaus, A., Hensley, M.L., Gathmann, I., Bolton, A.E., Hoomissen, I.C., Goldman, J.M. \& Radich, J.P. (2003) International Randomised Study of Interferon versus STI571 (IRIS) Study Group. Frequency of major molecular responses to imatinib or interferon a plus cytarabine in newly diagnosed chronic myeloid leukemia. N. Engl. J. Med., 349, 1423-1432.

Italian Cooperative Study Group on Chronic Myeloid Leukemia and Italian Group for Bone Marrow Transplantation. (1999) Monitoring treatment and survival in chronic myeloid leukemia. J. Clin. Oncol., 17, 1858-1868.

Kantarjian, H.M., Smith, T.L., O’Brien, S., Beran, M., Pierce, S. \& Talpaz, M. (1995) Prolonged survival in chronic myelogenous leukemia after cytogenetic response to interferon-alpha therapy. Ann. Intern. Med., 122, 254-261

Kantarjian, H., Sawyers, C., Hochhaus, A., Guilhot, F., Schiffer, C., Gambacorti-Passerini, C., Niederwieser, D., Resta, D., Capdeville, R., Zoellner, U., Talpaz, M., Druker, B., Goldman, J., O’Brien, S.G., Russell, N., Fischer, T., Ottmann, O., Cony-Makhoul, P., Facon, T., Stone, R., Miller, C., Tallman, M., Brown, R., Schuster, M., Loughran, T., Gratwohl, A., Mandelli, F., Saglio, G., Lazzarino, M., Russo, D., Baccarani, M. \& Morra, E.; International STI571 CML Study Group. (2002a) Hemato- logic and cytogenetic responses to imatinib mesylate in chronic myelogenous leukemia. N. Engl. J. Med., 346, 645-652.

Kantarjian, H.M., Talpaz, M., O’Brien, S., Smith, T.L., Giles, F.J., Faderl, S., Thomas, D.A., Garcia-Manero, G., Issa, J.P., Andreeff, M., Kornblau, S.M., Koller, C., Beran, M., Keating, M., Rios, M.B., Shan, J., Resta, D., Capdeville, R., Hayes, K., Albitar, M., Freireich, E.J. \& Cortes, J.E. (2002b) Imatinib mesylate for Philadelphia chromosome-positive, chronic-phase myeloid leukemia after failure of interferonalpha: follow-up results. Clin. Cancer Res., 8, 2177-2187.

Kantarjian, H.M., Cortes, J.E., O’Brien, S., Giles, F., GarciaManero, G., Faderl, S., Thomas, D., Jeha, S., Rios, M.B., Letvak, L., Bochinski, K., Arlinghaus, R. \& Talpaz, M. (2003) Imatinib mesylate therapy in newly diagnosed patients with Philadelphia chromosome-positive chronic myelogenous leukemia: high incidence of early complete and major cytogenetic responses. Blood, 101, 97-100.

Kantarjian, H., Talpaz, M., O’Brien, S., Garcia-Manero, G., Verstovsek, S., Giles, F., Rios, M.B., Shan, J., Letvak, L., Thomas, D., Faderl, S., Ferrajoli, A. \& Cortes, J. (2004) High-dose imatinib mesylate therapy in newly diagnosed Philadelphia chromosome-positive chronic phase chronic myeloid leukemia. Blood, 103, 2873-2878.

Lugo, T.G., Pendergast, A.M., Muller, A.J. \& Witte, O.N. (1990) Tyrosine kinase activity and transformation potency of bcr-abl oncogene products. Science, 247, 1079-1082.

Mauro, M.J., Druker, B.J. \& Maziarz, R.T. (2004) Divergent clinical outcome in two CML patients who discontinued imatinib therapy after achieving a molecular remission. Leuk Res., 28, Suppl. 1, S71-S73.

Merante, S., Orlandi, E., Bernasconi, P., Calatroni, S., Boni, M. \& Lazzarino, M. (2005) Outcome of four patients with chronic myeloid leukemia after imatinib mesylate discontinuation. Haematologica, 90, 979-981.

O’Brien, S.G., Guilhot, F., Larson, R.A., Gathmann, I., Baccarani, M., Cervantes, F., Cornelissen, J.J., Fischer, T., Hochhaus, A., Hughes, T., Lechner, K., Nielsen, J.L., Rousselot, P., Reiffers, J., Saglio, G., Shepherd, J., Simonsson, B., Gratwohl, A., Goldman, J.M., Kantarjian, H., Taylor, K., Verhoef, G., Bolton, A.E., Capdeville, R. \& Druker, B.J. (2003) IRIS Investigators. Imatinib compared with interferon and low-dose cytarabine for newly diagnosed chronic-phase chronic myeloid leukemia. N. Engl. J. Med., 348, 994-1004.

Olavarria, E., Kanfer, E., Szydlo, R., Kaeda, J., Rezvani, K., Cwynarski, K., Pocock, C., Dazzi, F., Craddock, C., Apperley, J.F., Cross, N.C. \& Goldman, J.M. (2001) Early detection of BCR-ABL transcripts by quantitative reverse transcriptase-polymerase chain reaction predicts outcome after allogeneic stem cell transplantation for chronic myeloid leukemia. Blood, 97, 1560-1565.

Paschka, P., Muller, M.C., Merx, K., Kreil, S., Schoch, C., Lahaye, T., Weisser, A., Petzold, A., Konig, H., Berger, U., Gschaidmeier, H., Hehlmann, R. \& Hochhaus, A. (2003) Molecular monitoring of response to imatinib (Glivec) in CML patients pretreated with interferon-alpha. Low levels of residual disease are associated with continuous remission. Leukemia, 17, 1687-1694.

Sawyers, C.L. (1999) Chronic myeloid leukemia. N. Engl. J. Med., 340, 1330-1340.

Schindler, T., Bornmann, W., Pellicena, P., Miller, W.T., Clarkson, B. \& Kuriyan, J. (2000) Structural mechanism for STI-571 inhibition of abelson tyrosine kinase. Science, 
289, 1938-1942.

Talpaz, M., Silver, R.T., Druker, B.J., Goldman, J.M., Gambacorti-Passerini, C., Guilhot, F., Schiffer, C.A., Fischer, T., Deininger, M.W., Lennard, A.L., Hochhaus, A., Ottmann, O.G., Gratwohl, A., Baccarani, M., Stone, R., Tura, S., Mahon, F.X., Fernandes-Reese, S., Gathmann, I., Capdeville, R., Kantarjian, H.M. \& Sawyers, C.L. (2002) Imatinib induces durable hematologic and cytogenetic responses in patients with accelerated phase chronic myeloid leukemia: results of a phase 2 study. Blood, 99,
1928-1937.

Yamada, M., Miyamura, K., Fujiwara, T., Yokoyama, H., Tomiya, Y., Ishizawa, K., Harigae, H., Kameoka, J. \& Sasaki, T. (2004) Imatinib mesylate in conjunction with allogeneic hematopoietic stem cell transplantation in patients with philadelphia chromosome positive leukemias: Report of 4 cases. Tohoku J. Exp. Med., 204, 79-84.

Yoshida, C. \& Melo, J.D. (2004) Biology of chronic myeloid leukemia and possible therapeutic approaches to imatinibresistant disease. Int. J. Hematol., 79, 420-433. 